PROCEEDINGS OF THE

AMERICAN MATHEMATICAL SOCIETY

Volume 135, Number 10, October 2007, Pages 3143-3150

S 0002-9939(07)08999-X

Article electronically published on June 19, 2007

\title{
CONVERGENCE OF ALMOST-ORBITS OF NONEXPANSIVE SEMIGROUPS IN BANACH SPACES
}

\author{
ANTHONY TO-MING LAU, KOJI NISHIURA, AND WATARU TAKAHASHI
}

(Communicated by Jonathan M. Borwein)

\begin{abstract}
The purpose of this paper is to show that the study of mean ergodic theorems for almost-orbits of semigroups of nonexpansive mappings on closed convex subsets of a Banach space can be reduced to the study of orbits for semigroups of nonexpansive mappings. This provides a unified approach to various mean ergodic theorems for almost-orbits in the literature and new applications.
\end{abstract}

\section{INTRODUCTION}

Let $C$ be a nonempty closed convex subset of a Banach space $E$. Then, a mapping $T$ of $C$ into itself is said to be nonexpansive if $\|T x-T y\| \leq\|x-y\|$ for every $x, y \in C$. Let $S$ be a semigroup and let $\mathcal{S}=\{T(t): t \in S\}$ be a representation of the semigroup $S$ as nonexpansive mappings from $C$ into itself, i.e., for each $t \in S$, $T(t)$ is a nonexpansive mapping of $C$ into itself and $T(s t)=T(s) T(t)$ for every $s, t \in S$. Then, a function $u: S \rightarrow C$ is said to be an almost-orbit of $\mathcal{S}$ if

$$
\inf _{w \in S} \sup _{s, t \in S}\|u(s w t)-T(s) u(w t)\|=0 .
$$

In this paper, we prove that mean ergodic theorems for almost-orbits of nonexpansive semigroups can be reduced to those for nonexpansive semigroups.

The notion of almost-orbit was first introduced by Kiuchi and Takahashi in 7 . Our approach unifies various mean ergodic theorems for almost-orbits already in the literature (see 7], 9], 10 and 13]) and gives new applications to results in [1], 2, 3], 5] and 6]. Baillon [4 proved the first nonlinear ergodic theorem for nonexpansive mappings in the framework of a Hilbert space: Let $C$ be a closed convex subset of a Hilbert space and let $T$ be a nonexpansive mapping of $C$ into itself. If the set $F(T)$ of fixed points of $T$ is nonempty, then for each $x \in C$, the Cesàro means $S_{n}(x)=\frac{1}{n} \sum_{k=0}^{n-1} T^{k} x$ converge weakly to some $y \in F(T)$. The notion of an almost-orbit for a nonexpansive mapping was introduced by Bruck in [5. It was extended by Miyadera and Kobayasi to the case of a one-parameter nonexpansive semigroup in [9] where they established the weak and strong almost

Received by the editors March 31, 2006.

2000 Mathematics Subject Classification. Primary 47H20; Secondary 47H09.

Key words and phrases. Mean ergodic theorem, nonexpansive semigroup, almost-orbit, regular net, invariant mean.

This research was supported by NSERC grant A-7679 and by Grant-in-Aid for General Scientific Research No. 15540157, the Ministry of Education, Science, Sports and Culture, Japan. 
convergence of such an almost-orbit in Banach spaces. These results were then extended to various forms by many authors; for example [6, 8, 10, 11, 13].

Our paper is organized as follows: in Section 2 we define some terminologies that we use; in Section 3 we prove our main results; in Section 4 we provide the applications of our results in Section 3.

\section{Preliminaries}

Let $E$ be a Banach space and let $S$ be a semigroup. We denote by $E^{*}$ the dual space of $E$ and by $\left\langle x, x^{*}\right\rangle$ the value of $x^{*} \in E^{*}$ at $x \in E$. We also denote by $B(S)$ the Banach space of all bounded real-valued functions on $S$ with supremum norm. For each $s \in S$ and $f \in B(S)$, we define elements $l_{s} f$ and $r_{s} f$ of $B(S)$ by $\left(l_{s} f\right)(t)=f(s t)$ and $\left(r_{s} f\right)(t)=f(t s)$ for all $t \in S$. We sometimes use $\mu_{t}(f(t))$ instead of $\mu(f)$ for $\mu \in B(S)^{*}$ and $f \in B(S)$.

Let $X$ be a subspace of $B(S)$ such that $1 \in X$ and $X$ is $l_{s^{-}}$and $r_{s}$-invariant, i.e., $l_{s}(X) \subset X$ and $r_{s}(X) \subset X$ for every $s \in S$. Then, a net $\left\{\mu_{\alpha}\right\}_{\alpha \in I}$ of continuous linear functionals on $X$ is said to be strongly regular 6 if it satisfies the following conditions:

(1) $\sup _{\alpha}\left\|\mu_{\alpha}\right\|<\infty$;

(2) $\lim _{\alpha} \mu_{\alpha}(1)=1$;

(3) $\lim _{\alpha}\left\|\mu_{\alpha}-l_{s}^{*} \mu_{\alpha}\right\|=0$ and $\lim _{\alpha}\left\|\mu_{\alpha}-r_{s}^{*} \mu_{\alpha}\right\|=0$ for every $s \in S$.

We say that $\left\{\mu_{\alpha}\right\}_{\alpha \in I}$ is weak ${ }^{*}$ regular if (3) is replaced by the weaker condition:

(4) weak $^{*}-\lim _{\alpha}\left(\mu_{\alpha}-l_{s}^{*} \mu_{\alpha}\right)=0$ and weak ${ }^{*}-\lim _{\alpha}\left(\mu_{\alpha}-r_{s}^{*} \mu_{\alpha}\right)=0$ for every $s \in S$.

Let $f$ be a function of $S$ into $E$ such that $\{f(t): t \in S\}$ is relatively weakly compact and let $X$ be a subspace of $B(S)$ such that for each $x^{*} \in E^{*}$, the function $t \mapsto\left\langle f(t), x^{*}\right\rangle$ is an element of $X$. Then, for any $\mu \in X^{*}$ there exists a unique element $f_{\mu}$ in $E$ such that

$$
\mu_{t}\left\langle f(t), x^{*}\right\rangle=\left\langle f_{\mu}, x^{*}\right\rangle
$$

for every $x^{*} \in E^{*}$; see [12, 6]. Following [6], we write such $f_{\mu}$ by $\int f(t) d \mu(t)$.

Let $C$ be a nonempty closed convex subset of $E$. Let $\mathcal{S}=\{T(t): t \in S\}$ be a representation of the semigroup $S$ as nonexpansive mappings from $C$ into itself and let $X$ be a subspace of $B(S)$. Then, a function $u: S \rightarrow C$ is said to be a nearly almost-orbit of $\mathcal{S}$ if

$$
\inf _{s \in S} \sup _{t \in S}\|u(t s)-T(t) u(s)\|=0
$$

Clearly, every almost-orbit of $\mathcal{S}$ is a nearly almost-orbit. We denote by $N A O(\mathcal{S}, X)$ the set of all nearly almost-orbits $u$ of $\mathcal{S}$ such that $\{u(t): t \in S\}$ is relatively weakly compact and for each $x^{*} \in E^{*}$, the function $t \mapsto\left\langle u(t), x^{*}\right\rangle$ is an element of $X$.

\section{MAIN RESUlts}

First we show the following lemma.

Lemma 3.1. Let $E$ be a Banach space and let $S$ be a semigroup. Let $f$ be a function of $S$ into $E$ such that $\{f(t): t \in S\}$ is relatively weakly compact and let $X$ be a subspace of $B(S)$ such that $1 \in X$, for each $x^{*} \in E^{*}$, the function $t \mapsto\left\langle f(t), x^{*}\right\rangle$

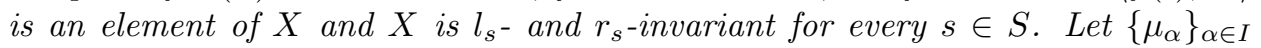
be a weak ${ }^{*}$ regular net of continuous linear functionals on $X$. If for each $h \in S$, 
$\int f(h t) d \mu_{\alpha}(t)$ converges weakly to some $y_{h} \in E$, then there exists $\mu \in X^{*}$ such that $\|\mu\| \leq K$,

$$
\mu\left(l_{s} g\right)=\mu\left(r_{s} g\right)=\mu(g),
$$

for every $g \in X$ and $s \in S$, and

$$
y_{h}=\int f(t) d \mu(t)
$$

for every $h \in S$, where $K=\sup _{\alpha \in I}\left\|\mu_{\alpha}\right\|$.

Proof. Assume that for each $h \in S, \int f(h t) d \mu_{\alpha}(t)$ converges weakly to some $y_{h} \in E$. Let $B=\left\{\mu \in X^{*}:\|\mu\| \leq K\right\}$. Since $B$ is compact in the weak* topology, there exists a subnet $\left\{\mu_{\alpha_{\beta}}\right\}_{\beta \in J}$ of $\left\{\mu_{\alpha}\right\}_{\alpha \in I}$ such that $\mu_{\alpha_{\beta}}$ converges to some $\mu \in B$ in the weak* topology. Let $\varepsilon>0, g \in X$ and $s \in S$. Then, there exists $\alpha_{0} \in I$ such that $\left|\mu_{\alpha}\left(l_{s} g\right)-\mu_{\alpha}(g)\right|<\varepsilon$ for every $\alpha \geq \alpha_{0}$. Since $\mu_{\alpha_{\beta}}$ converges to $\mu \in B$ in the weak* topology, we can choose $\alpha_{1} \geq \alpha_{0}$ such that $\left|\mu_{\alpha_{1}}(g)-\mu(g)\right|<\varepsilon$ and $\left|\mu_{\alpha_{1}}\left(l_{s} g\right)-\mu\left(l_{s} g\right)\right|<\varepsilon$. So, we have

$$
\begin{aligned}
& \left|\mu\left(l_{s} g\right)-\mu(g)\right| \\
& \quad \leq\left|\mu\left(l_{s} g\right)-\mu_{\alpha_{1}}\left(l_{s} g\right)\right|+\left|\mu_{\alpha_{1}}\left(l_{s} g\right)-\mu_{\alpha_{1}}(g)\right|+\left|\mu_{\alpha_{1}}(g)-\mu(g)\right| \\
& \quad<\varepsilon+\varepsilon+\varepsilon=3 \varepsilon .
\end{aligned}
$$

Since $\varepsilon>0$ is arbitrary, we have $\mu\left(l_{s} g\right)=\mu(g)$. Similarly, we have $\mu\left(r_{s} g\right)=\mu(g)$. Since $\mu_{\alpha_{\beta}}$ converges to $\mu \in B$ in the weak* topology, it follows that for each $h \in S$, $\int f(h t) d \mu_{\alpha_{\beta}}(t)$ converges weakly to $\int f(h t) d \mu(t)$. Therefore, we obtain

$$
y_{h}=\int f(h t) d \mu(t)=\int f(t) d \mu(t)
$$

for every $h \in S$.

Now we prove the main theorems of this paper.

Theorem 3.2. Let $C$ be a nonempty closed convex subset of a Banach space E. Let $S$ be a semigroup and let $\mathcal{S}=\{T(t): t \in S\}$ be a representation of the semigroup $S$ as nonexpansive mappings from $C$ into itself such that for each $x \in C,\{T(t) x$ : $t \in S\}$ is relatively weakly compact. Let $X$ be a subspace of $B(S)$ such that $1 \in X$, for each $x \in C$ and $x^{*} \in E^{*}$, the function $t \mapsto\left\langle T(t) x, x^{*}\right\rangle$ is an element of $X$ and $X$ is $l_{s^{-}}$and $r_{s^{-}}$invariant for every $s \in S$. Let $\left\{\mu_{\alpha}\right\}_{\alpha \in I}$ be a strongly regular net of continuous linear functionals on $X$. Then the following are equivalent:

(1) For each $x \in C, \int T(h t) x d \mu_{\alpha}(t)$ weakly converges uniformly in $h \in S$.

(2) For each $u \in N A O(\mathcal{S}, X), \int u(h t) d \mu_{\alpha}(t)$ weakly converges uniformly in $h \in S$.

Proof. Since $(2) \Rightarrow(1)$ clearly holds, it is sufficient to show $(1) \Rightarrow(2)$. Let $u \in N A O(\mathcal{S}, X)$ and $\varepsilon>0$. Since $u$ is a nearly almost-orbit $\mathcal{S}$, there exists $s_{0} \in S$ such that

$$
\sup _{t \in S}\left\|u\left(t s_{0}\right)-T(t) u\left(s_{0}\right)\right\|<\varepsilon .
$$

Let $K=\sup _{\alpha \in I}\left\|\mu_{\alpha}\right\|$. From the hypothesis and Lemma 3.1, there exists $\mu \in X^{*}$ such that $\|\mu\| \leq K, \mu\left(l_{s} g\right)=\mu\left(r_{s} g\right)=\mu(g)$ for every $g \in X$ and $s \in S$ and $\int T(h t) u\left(s_{0}\right) d \mu_{\alpha}(t)$ converges weakly to $\int T(t) u\left(s_{0}\right) d \mu(t)$ uniformly in $h \in S$. We 
show that $\int u(h t) d \mu_{\alpha}(t)$ converges weakly to $\int u(t) d \mu(t)$ uniformly in $h \in S$. By (3.1), we have

$$
\begin{aligned}
\left\|\int u(t) d \mu(t)-\int T(t) u\left(s_{0}\right) d \mu(t)\right\| & =\left\|\int u\left(t s_{0}\right) d \mu(t)-\int T(t) u\left(s_{0}\right) d \mu(t)\right\| \\
& \leq\|\mu\| \sup _{t \in S}\left\|u\left(t s_{0}\right)-T(t) u\left(s_{0}\right)\right\| \\
& \leq K \varepsilon
\end{aligned}
$$

and for each $\alpha$ and $h \in S$,

$$
\begin{aligned}
& \left\|\int u\left(h t s_{0}\right) d \mu_{\alpha}(t)-\int T(h t) u\left(s_{0}\right) d \mu_{\alpha}(t)\right\| \\
& \quad \leq\left\|\mu_{\alpha}\right\| \sup _{t \in S}\left\|u\left(h t s_{0}\right)-T(h t) u\left(s_{0}\right)\right\| \\
& \quad \leq K \varepsilon .
\end{aligned}
$$

Since $\left\{\mu_{\alpha}\right\}_{\alpha \in I}$ is strongly regular, there exists $\alpha_{0}$ such that for each $\alpha \geq \alpha_{0}$,

$$
\left\|\mu_{\alpha}-r_{s_{0}}^{*} \mu_{\alpha}\right\|<\varepsilon
$$

Let $M=\sup _{t \in S}\|u(t)\|$. By (3.4), we have for each $\alpha \geq \alpha_{0}$ and $h \in S$,

$$
\begin{aligned}
& \left\|\int u(h t) d \mu_{\alpha}(t)-\int u\left(h t s_{0}\right) d \mu_{\alpha}(t)\right\| \\
& =\left\|\int u(h t) d \mu_{\alpha}(t)-\int u(h t) d\left(r\left(s_{0}\right)^{*} \mu_{\alpha}\right)(t)\right\| \\
& \quad \leq\left\|\mu_{\alpha}-r_{s_{0}}^{*} \mu_{\alpha}\right\| \sup _{t \in S}\|u(h t)\| \\
& \quad \leq M \varepsilon .
\end{aligned}
$$

Let $x^{*} \in E^{*}$ with $\left\|x^{*}\right\| \leq 1$. In the case of weak convergence, there exists $\alpha_{1} \geq \alpha_{0}$ such that for each $\alpha \geq \alpha_{1}$,

$$
\left|\left\langle\int T(h t) u\left(s_{0}\right) d \mu_{\alpha}(t)-\int T(t) u\left(s_{0}\right) d \mu(t), x^{*}\right\rangle\right|<\varepsilon .
$$

From (3.2), (3.3), (3.5) and (3.6), we obtain for each $\alpha \geq \alpha_{1}$ and $h \in S$,

$$
\begin{aligned}
&\left|\left\langle\int u(h t) d \mu_{\alpha}(t)-\int u(t) d \mu(t), x^{*}\right\rangle\right| \\
& \leq \mid\left\langle\int u(h t) d \mu_{\alpha}(t)-\int u\left(h t s_{0}\right) d \mu_{\alpha}(t), x^{*}\right\rangle \mid \\
&+\left|\left\langle\int u\left(h t s_{0}\right) d \mu_{\alpha}(t)-\int T(h t) u\left(s_{0}\right) d \mu_{\alpha}(t), x^{*}\right\rangle\right| \\
&+\left|\left\langle\int T(h t) u\left(s_{0}\right) d \mu_{\alpha}(t)-\int T(t) u\left(s_{0}\right) d \mu(t), x^{*}\right\rangle\right| \\
&+\left|\left\langle\int T(t) u\left(s_{0}\right) d \mu(t)-\int u(t) d \mu(t), x^{*}\right\rangle\right| \\
& \leq \quad(M+2 K+1) \varepsilon .
\end{aligned}
$$

Since $\varepsilon>0$ is arbitrary, we obtain the conclusion. 
Remark 3.3. Theorem 3.2 also holds when "weakly" is replaced by "strongly". Indeed, if for each $x \in C, \int T(h t) x d \mu_{\alpha}(t)$ strongly converges uniformly in $h \in S$, then there exists $\alpha_{2} \geq \alpha_{0}$ such that for each $\alpha \geq \alpha_{2}$,

$$
\left\|\int T(h t) u\left(s_{0}\right) d \mu_{\alpha}(t)-\int T(t) u\left(s_{0}\right) d \mu(t)\right\|<\varepsilon .
$$

From (3.2), (3.3), (3.5) and (3.7), we obtain for each $\alpha \geq \alpha_{2}$ and $h \in S$,

$$
\begin{aligned}
& \left\|\int u(h t) d \mu_{\alpha}(t)-\int u(t) d \mu(t)\right\| \\
& \leq \quad\left\|\int u(h t) d \mu_{\alpha}(t)-\int u\left(h t s_{0}\right) d \mu_{\alpha}(t)\right\| \\
& \quad+\left\|\int u\left(h t s_{0}\right) d \mu_{\alpha}(t)-\int T(h t) u\left(s_{0}\right) d \mu_{\alpha}(t)\right\| \\
& \quad+\left\|\int T(h t) u\left(s_{0}\right) d \mu_{\alpha}(t)-\int T(t) u\left(s_{0}\right) d \mu(t)\right\| \\
& \quad+\left\|\int T(t) u\left(s_{0}\right) d \mu(t)-\int u(t) d \mu(t)\right\| \\
& \leq \quad(M+2 K+1) \varepsilon .
\end{aligned}
$$

Since $\varepsilon>0$ is arbitrary, this implies that $\int u(h t) d \mu_{\alpha}(t)$ converges strongly to $\int u(t) d \mu(t)$ uniformly in $h \in S$.

Concerning Theorems 3.2 and Lemma 3.1, we provide the following result.

Theorem 3.4. Let $C$ be a nonempty closed convex subset of a Banach space $E$. Let $S$ be a semigroup and let $\mathcal{S}=\{T(t): t \in S\}$ be a representation of the semigroup $S$ as nonexpansive mappings from $C$ into itself such that for each $x \in C,\{T(t) x: t \in$ $S\}$ is relatively weakly compact. Let $X$ be a subspace of $B(S)$ such that $1 \in X$, for each $x \in C$ and $x^{*} \in E^{*}$, the function $t \mapsto\left\langle T(t) x, x^{*}\right\rangle$ is an element of $X$ and $X$ is $r_{s}$-invariant for every $s \in S$. Let $\mu$ be an element of $X^{*}$ such that $\mu(r(s) f)=\mu(f)$ for every $f \in X$ and $s \in S$. Then the following are equivalent:

(1) For each $x \in C, \int T(t) x d \mu(t) \in F(\mathcal{S})$.

(2) For each $u \in N A O(\mathcal{S}, X), \int u(t) d \mu(t) \in F(\mathcal{S})$.

Proof. Since $(2) \Rightarrow(1)$ clearly holds, it is sufficient to show $(1) \Rightarrow(2)$. Let $u \in N A O(\mathcal{S}, X)$ and $\varepsilon>0$. As in the proof of Theorem 3.2. we have for some $s_{0} \in S$,

$$
\left\|\int u(t) d \mu(t)-\int T(t) u\left(s_{0}\right) d \mu(t)\right\| \leq \varepsilon
$$

So, we have for each $s \in S$,

$$
\begin{aligned}
\| & T(s) \int u(t) d \mu(t)-\int u(t) d \mu(t) \| \\
& \leq\left\|T(s) \int u(t) d \mu(t)-\int T(t) u\left(s_{0}\right) d \mu(t)\right\|+\left\|\int T(t) u\left(s_{0}\right) d \mu(t)-\int u(t) d \mu(t)\right\| \\
& \leq 2\left\|\int u(t) d \mu(t)-\int T(t) u\left(s_{0}\right) d \mu(t)\right\| \\
& <2 \varepsilon .
\end{aligned}
$$

Since $\varepsilon>0$ is arbitrary, we obtain $\int u(t) d \mu(t) \in F(\mathcal{S})$. 


\section{Applications}

In this section, by using Theorems 3.2 and 3.4, we provide some colloraries. From [5] and [6], we obtain the following results of weak almost convergence.

Corollary 4.1. Let $C$ be a nonempty closed convex subset of a uniformly convex Banach space $E$ whose norm is Fréchet differentiable. Let $T$ be a nonexpansive mapping of $C$ into itself such that $F(T)$ is nonempty. Then for each nearly almostorbit $\left\{x_{n}\right\}$ of $T, \frac{1}{n} \sum_{i=0}^{n-1} x_{i+h}$ converges weakly to some $y \in F(T)$ uniformly in $h=0,1,2, \ldots$

Corollary 4.2. Let $C$ and $E$ be as in Corollary 4.1. Let $\mathcal{S}=\{T(t): t \geq 0\}$ be a one-parameter nonexpansive semigroup on $C$ such that $F(\mathcal{S})$ is nonempty. Then for each continuous nearly almost-orbit $u$ of $\mathcal{S}, \frac{1}{t} \int_{0}^{t} u(h+s) d s$ converges weakly to some $y \in F(\mathcal{S})$ uniformly in $h \geq 0$.

Corollary 4.3. Let $C$ and $E$ be as in Corollary 4.1. Let $S$ be a commutative semigroup and let $\mathcal{S}=\{T(t): t \in S\}$ be a representation of the semigroup $S$ as nonexpansive mappings from $C$ into itself such that $F(\mathcal{S})$ is nonempty. Let $X$ be a subspace of $B(S)$ such that $1 \in X$, for each $x \in C$ and $x^{*} \in E^{*}$, the function $t \mapsto\left\langle T(t) x, x^{*}\right\rangle$ is an element of $X$ and $X$ is $r_{s}$-invariant for every $s \in S$. Let $\left\{\mu_{\alpha}\right\}_{\alpha \in I}$ be a strongly regular net of continuous linear functionals on $X$. Then for each $u \in N A O(\mathcal{S}, X), \int u(h t) d \mu_{\alpha}(t)$ converges weakly to some $y \in F(\mathcal{S})$ uniformly in $h \in S$.

From [2], 3] and [1], we obtain the following results of strong almost convergence.

Corollary 4.4. Let $C$ be a nonempty closed convex subset of a strictly convex Banach space $E$. Let $T$ be a nonexpansive mapping of $C$ into itself such that $T(C)$ is relatively compact. Then for each nearly almost-orbit $\left\{x_{n}\right\}$ of $T, \frac{1}{n} \sum_{i=0}^{n-1} x_{i+h}$ converges strongly to some $y \in F(T)$ uniformly in $h=0,1,2, \ldots$

Corollary 4.5. Let $E$ be as in Corollary 4.4 and let $C$ be a nonempty compact convex subset of $E$. Let $\mathcal{S}=\{T(t): t \geq 0\}$ be a one-parameter nonexpansive semigroup on $C$. Then for each continuous nearly almost-orbit $u$ of $\mathcal{S}$, $\frac{1}{t} \int_{0}^{t} u(h+s) d s$ converges strongly to some $y \in F(\mathcal{S})$ uniformly in $h \geq 0$.

Corollary 4.6. Let $C$ and $E$ be as in Corollary 4.4, Let $S$ be a commutative semigroup and let $\mathcal{S}=\{T(t): t \in S\}$ be a representation of the semigroup $S$ as nonexpansive mappings from $C$ into itself such that $\bigcup_{t \in S} T(t)(C) \subset K$ for some compact subset $K$ of $C$. Let $X$ be a subspace of $B(S)$ such that $1 \in X$, for each $x \in C$ and $x^{*} \in E^{*}$, the function $t \mapsto\left\langle T(t) x, x^{*}\right\rangle$ is an element of $X$ and $X$ is $r_{s}$-invariant for every $s \in S$. Let $\left\{\mu_{\alpha}\right\}_{\alpha \in I}$ be a strongly regular net of continuous linear functionals on $X$. Then for each $u \in N A O(\mathcal{S}, X), \int u(h t) d \mu_{\alpha}(t)$ converges strongly to some $y \in F(\mathcal{S})$ uniformly in $h \in S$.

\section{EXAMPles AND REMARKS}

In this section, we give examples and remarks concerning our results.

Remark 5.1. (1) If $m$ is an invariant mean on $B(S)$, i.e., $m$ is a translation invariant norm one positive linear functional on $B(S)$, then $\{m\}$ is strongly regular. 
(2) If $m$ is an invariant mean on $B(S)$, then there exists a net of means with finite support, i.e., $\mu_{\alpha}=\sum_{i=1}^{n} \lambda_{i} \delta_{a_{i}}$ (convex combination of point evaluations) such that weak ${ }^{*}-\lim _{\alpha} \mu_{\alpha}=m$. In particular, the net $\left\{\mu_{\alpha}\right\}$ is weak ${ }^{*}$ regular. Consequently, weak ${ }^{*}$ regular nets with finite supports always exist when $S$ is commutative, or more generally when $B(S)$ has an invariant mean.

Example $5.2([1])$. Let $\mathcal{S}=(\{0,1,2, \cdots\},+)$ and $\mu_{n}=\frac{1}{n} \sum_{k=0}^{n-1} \delta_{k}$. Then, $\left\{\mu_{n}\right\}$ is a weak $^{*}$ regular sequence of finite means on $\mathcal{S}$. In particular, if $T$ is any nonexpansive mapping from a nonempty convex subset $C$ of a Hilbert space $H$ into $C$, then the sequence of mappings associated to $\mu_{n}$ is given by $T_{\mu_{n}}=\frac{1}{n} \sum_{k=0}^{n-1} T^{k}$, where $T^{0}=I$. Suppose the fixed point set for $T$ in $C$ is nonempty. Then by a result of Rodé in 11], the sequence $T_{\mu_{n}} x$ converges weakly to a fixed point for $T$. Corollary 4.1 is an improvement of Rodé's result. This also gives Corollary 4.4

Example 5.3. Let $S=[0, \infty), \mathcal{S}=\{S(t): t \geq 0\}$ and let $X$ be the Banach space $C B(S)$ of all bounded continuous functions on $S$ with supremum norm. Define

$$
\lambda_{s}(f)=\frac{1}{s} \int_{0}^{s} f(t) d t
$$

for every $s>0$ and $f \in X$. Then, we obtain

$$
\begin{aligned}
\left\|\lambda_{s}-r_{k}^{*} \lambda_{s}\right\| & =\sup _{\|f\| \leq 1}\left\|\frac{1}{s} \int_{0}^{s} f(t) d t-\frac{1}{s} \int_{0}^{s} f(t+k) d t\right\| \\
& =\frac{1}{s} \sup _{\|f\| \leq 1}\left\|\int_{0}^{s} f(t) d t-\int_{k}^{s+k} f(t) d t\right\| \\
& =\frac{1}{s} \sup _{\|f\| \leq 1}\left\|\int_{0}^{k} f(t) d t-\int_{s}^{s+k} f(t) d t\right\| \\
& \leq \frac{1}{s} \sup _{\|f\| \leq 1}\left(\int_{0}^{k}\|f(t)\| d t+\int_{s}^{s+k}\|f(t)\| d t\right) \\
& =\frac{2 k}{s} \rightarrow 0,
\end{aligned}
$$

as $s \rightarrow \infty$. In particular, $\left\{\lambda_{s} ; s>0\right\}$ is a strongly regular net of means on $X$. This gives Corollary 4.2 and Corollary 4.5 .

Example $5.4([7)$. Consider the initial value problem:

$$
\begin{gathered}
\frac{d u(t)}{d t}+A u(t) \ni f(t), t>0, \\
u(0)=x,
\end{gathered}
$$

where $A$ is an $m$-accretive operator in $E, f \in L^{1}(0, \infty ; E)$ and $x \in \overline{D(A)}$. Then it is well known that (5.1) has a unique integral solution [7] and the integral solution $u(t)$ of (5.1) is an almost-orbit of a one-parameter nonexpansive semigroup on $\overline{D(A)}$ generated by $-A$.

Remark 5.5. Our main results (Theorem 3.2 and Theorem 3.4) are new even for a semigroup generated by a nonexpansive mapping (Corollary 4.1) and for a oneparameter semigroup (Corollary 4.2). 
We thank the referee for his/her careful reading of the paper and his/her many valuable suggestions.

\section{REFERENCES}

[1] S. Atsushiba A. T. Lau and W. Takahashi, Nonlinear strong ergodic theorems for commutative nonexpansive semigroups on strictly convex Banach spaces, J. Nonlinear and Convex Anal., 1 (2000), 213-231. MR.1777140 (2002d:47077)

[2] S. Atsushiba and W. Takahashi, A nonlinear strong ergodic theorem for nonexpansive mappings with compact domains, Math. Japon., 52 (2000), 183-195. MR1793266 (2001i:47089)

[3] S. Atsushiba and W. Takahashi, Strong convergence theorems for one-parameter nonexpansive semigroups with compact domains, Fixed Point Theory and Applications (Y. J. Cho, J. K. Kim and S. M. Kang, Eds.), Nova Science Publisher, Inc., Huntington New York (2002), 15-31. MR2081406 (2005i:47081)

[4] J. B. Baillon, Un théorème de type ergodique pour les contractions non linéaires dans un espace de Hilbert, C. R. Acad. Sci. Paris Sér. A-B, 280 (1975), 1511-1514. MR0375009 $(51: 11205)$

[5] R. E. Bruck, A simple proof of the mean ergodic theorem for nonlinear contractions in Banach spaces, Israel J. Math., 32 (1979), 107-116. MR531254 (80j:47066)

[6] N. Hirano, K. Kido and W. Takahashi, Nonexpansive retractions and nonlinear ergodic theorems in Banach spaces, Nonlinear Anal., 12 (1988), 1269-1281. MR.969505 (90a:47138)

[7] H. Kiuchi and W. Takahashi, Asymptotic behavior of almost-orbits of nonexpansive semigroups without convexity, Kodai Math. J., 15 (1992), 185-192. MR1185417 (93j:47092)

[8] A. T. Lau, N. Shioji and W. Takahashi, Existence of nonexpansive retractions for amenable semigroups of nonexpansive mappings and nonlinear ergodic theorems in Banach spaces, J. Funct. Anal., 161 (1999), 62-75. MR.1670206 (99j:47079)

[9] I. Miyadera and K. Kobayasi, On the asymptotic behaviour of almost-orbits of nonlinear contraction semigroups in Banach spaces, Nonlinear Anal., 6 (1982), 349-365. MR654811 (84c:47064)

[10] H. Oka, Nonlinear ergodic theorems for commutative semigroups of asymptotically nonexpansive mappings, Nonlinear Anal., 18 (1992), 619-635. MR.1157563 (93d:47126)

[11] G. Rodé, An ergodic theorem for semigroups of nonexpansive mappings in a Hilbert space, J. Math. Anal. Appl., 85 (1982), 172-178. MR647565 (83k:47038)

[12] W. Takahashi, A nonlinear ergodic theorem for an amenable semigroup of nonexpansive mappings in a Hilbert space, Proc. Amer. Math. Soc., 81 (1981), 253-256. MR593468 (82f:47079)

[13] W. Takahashi and J. Y. Park, On the asymptotic behavior of almost-orbits of commutative semigroups in Banach spaces, Nonlinear and Convex Analysis, Marcel Dekker, Inc., New York and Basel (1987), 271-293. MR892798 (88g:47131)

Department of Mathematical and Satistical Sciences, University of Albert, Edmonton, Alberta, Canada T6G 2G1

E-mail address: tlau@math.ualberta.ca

Department of General Education, Fukushima national College of Technology, TAIRA, IWAKI-SHI 970-8034, JAPAN

E-mail address: nishiura@fukushima-nct.ac.jp

Department of Mathematical and Computing Sciences, Tokyo Institute of Technology, Oh-OKayama, Meguro-Ku, TOKyo 152-8552, Japan

E-mail address: wataru@is.titech.ac.jp 